\title{
Article \\ Attaining Ultraprecision Machining by Feed Drive System Stability Control with Piezoelectric Preloading Actuators
}

\author{
Guangcheng Zhang ${ }^{1,2, * \mathbb{C}}$, Hao Jin ${ }^{1}$ and Yueh-Jaw Lin $^{2}$ \\ 1 School of Mechanical Engineering, University of Shanghai for Science and Technology, \\ Shanghai 200093, China; 193711474@st.usst.edu.cn \\ 2 College of Engineering and Engineering Technology, Northern Illinois University, DeKalb, IL 60115, USA; \\ ylin1@niu.edu \\ * Correspondence: g.c.zhang@usst.edu.cn
}

Citation: Zhang, G.; Jin, H.; Lin, Y.-J Attaining Ultraprecision Machining by Feed Drive System Stability Control with Piezoelectric Preloading Actuators. Appl. Sci. 2021, 11, 8491. https://doi.org/10.3390/app11188491

Academic Editor: John D. Clayton

Received: 25 August 2021

Accepted: 9 September 2021

Published: 13 September 2021

Publisher's Note: MDPI stays neutral with regard to jurisdictional claims in published maps and institutional affiliations.

Copyright: (c) 2021 by the authors. Licensee MDPI, Basel, Switzerland. This article is an open access article distributed under the terms and conditions of the Creative Commons Attribution (CC BY) license (https:// creativecommons.org/licenses/by/ $4.0 /)$.

\begin{abstract}
In this paper, a variable preload force control structure utilizing piezoelectric actuators (PEAs) is proposed for the stability control of the feed drive system. Three PEAs are installed between the two nuts to exert preload force on the ball screw, leading to an elimination or substantial reduction of the backlash, which is the main cause of instability of feed drives. This results in better machining precision throughout the operation process. In addition, the force analysis of the whole preload feed drive system is established. Moreover, the hysteresis of the PEAs is determined with reference to the Prandtl-Ishlinskii (P-I) model. Lastly, the P-I model-based feedforward controller is applied to the feed drive system to improve the resultant machining precision. Based on the modeling and experiments, to demonstrate the efficacy and high-performance of the proposed P-I model-based control algorithm against conventional PID control system, comparative experiments are conducted, showing satisfactory results.
\end{abstract}

Keywords: feed drive system; stability control; piezoelectric; ultraprecision machining

\section{Introduction}

Ultraprecision computer numerical control (CNC) machines, as cutting-edge equipment in the manufacturing industry, have been attracting lots of attention in recent years. However, chatter, a self-excited vibration occurring during the machining process, often causes poor surface quality, higher inaccuracy, and undesirable tool damage [1]. This has motivated many machining researchers over the years to delve into the development of methodologies that warrant the use of chatter suppression high-performance spindles in $\mathrm{CNC}$ machines [2], while also improving the stability of the feed system during machining operations.

At present, feed systems can be divided into two main types, one is directly powered by the liner motor, the other uses a rotating motor through a ball screw and a nut to drive the system [3]. Among machine tools, ball screw drives have been widely applied for realizing linear motion due to their advantages, which include high transmission efficiency, high rigidity, strong anti-interference capability, etc. [4]. They have become irreplaceable transmission devices in high-end CNC machine tools. Nevertheless, there is a backlash between the ball screw and the nut, and because of this, vibration will be caused and the stiffness of the ball screw is not high enough to achieve high machining accuracy [5]. The backlash should be reduced or eliminated, and this goal is normally achieved through the application of a preload force.

With respect to the application of preload force in machine tools, there have been plenty of studies addressing this. In the practical applications, springs are commonly applied in the system to generate a constant preload force. However, the preload force introduced by the above method is not adjustable, limiting the application of the technology. Considering the development of the variable preload force technology, the existing preload mechanisms 
can be categorized into passive control devices and active control devices on the basis of their control principle [6]. With regard to passive devices, V Paleu et al. [7] developed the application of Fe-Mn-Si-based shape memory alloys for axial variable preload force of ball bearings. Dong Hyeon Kim et al. [8] used an eccentric mass device to convert the radial force caused by the centrifugal force into axial force in order to apply the preload force. However, these devices are still unable to achieve arbitrary adjustment of the preload force. Regarding active control devices, Gavrilin et al. [9] applied preload force to eliminate the backlash and enhance the stiffness. Hongrui Cao et al. [10] used a hydraulic system to exert preload force, and this was able to generate huge force. However, the hydraulic system also had limitations that the equipment structure was complex, the size was relatively large, and the response was is slow [11].

The methods described above introduce a preload force onto the bearing of the spindles. When it comes to milling machines, the ball screw directly affects the quality of the processing owing to the additional presence of backlash. To solve this problem, preload force also needs to be loaded onto the ball screw.

In the traditional method, considering a single nut, the great steel ball method, whereby the groove is smaller than the steel ball, is used to achieve preload. The stretching method, in double-nut cases, refers to a technique whereby the two nuts are separated by a thick washer squeezed between them [12]. The above methods do not have the feature of dynamic adjustment and cannot meet the requirement of high precision. In [13], a device was developed using an electromagnet to generate the preload force. However, the size of the magnet, magnetic isolation, and eddy current heating problems hinder its application in engineering practice. In [14], a cylindrical giant magneto strictive actuator was used to exert preload force on a ball screw. The drive coil generates lots of heat, so a cooling system is needed, resulting in large size and heavy mass. A type of ball screw preload structure using PEAs was introduced in [15], which achieved real-time adjustment of preload force, but the response was not fast enough due to the control strategy used.

In the field of high-accuracy control, PEAs are used extensively and have a promising development application prospect. Due to the characteristics of fast response, large mass ratio, and high stiffness [16], the PEAs have been widely applied in micro robots [17], scanning tunneling microscopes [18], etc. Consequently, preload force control with rapid response speed can be achieved through PEAs. Meanwhile, the inherent nonlinear property of PEAs, coming from the creep, hysteresis and vibration of the piezoelectric $[19,20]$, greatly decreases the control precision of PEAs.

Among the three characteristics mentioned above, hysteresis has the predominant influence on control performance. Hysteresis, which has a major impact on the nonlinear characteristic of PEAs, describes the dependence of the state of a system on its history, as occurs in shape-memory alloys and piezoelectric material. Due to their hysteretic character, both current and historical control inputs affect the output of PEAs.

For decades, researchers have made efforts to eliminate the nonlinearity of PEAs. There have been many theoretical studies on hysteresis, and the most popular method for compensating the hysteresis error is inversion-based feedforward control. Before applying this method, a model of hysteresis is needed. Simply speaking, two typical categories of hysteresis models, namely, physics-based models and phenomenological models [21], have been proposed in the literature. Among physics-based models, the Jiles-Atherton model is a well-known model [22], and the Domain Wall Model [23] is based on this. The inversion model of these two models is available for linear controller design. One non-negligible limitation of physics-based models is that they are generally quite complicated.

With respect to phenomenological models, the classical Preisach model is described by combining an infinite number of Preisach hysteresis operators, and it is widely considered to be a base toolkit of hysteresis modeling due to its general structure [24]. The nonlinear Preisach model has a broader area of application over the classical one, and makes it possible to fit experimentally measured data using first- and second-order transition curves [25]. Another widely used model is the Prandtl-Ishlinskii (P-I) model, where 
hysteresis is modeled by the elementary hysteresis operator called Play or Stop. The analytical inverse of P-I model is available. Consequently, it is more efficient for real-time applications [26]. Furthermore, there exist many other models and they are also pertinent for use in hysteresis. All in all, the aforementioned models should be inverted without exception so that they can compensate the hysteresis error.

In this paper, a variable preload force control device using three PEAs is developed, and a P-I model-based control strategy for realizing the precise control of the output force is applied. PEAs are used to increase the stability of feed drive system, considering the application of PEAs on preload system, the force control is relatively more simple and convenient way. Three PEAs are installed between the double-nut to exert preload force on the ball screw and eliminate the backlash. Furthermore, the main idea of the control method is to use a feedforward compensator associated with a PID controller. Thereby, the inverse of the P-I model can be readily applied as the compensator. After conducting a series of experiments, the feedforward compensator along with the PID controller attained better performance compared with the one using a traditional PID controller. This paper focuses on the increased stability of the feed drive system in ultraprecision machining with the application of PEAs and the P-I model-based control strategy to increase the stiffness of the system at a high response rate.

\section{Structure and Analysis of Automatic Adjustable Preload System}

\subsection{Structure and Operating Principle of Automatic Adjustable Preload System}

For the purpose of realizing variable (automatically adjustable) preload force control, a system using PEAs is proposed. The schematic diagram of the variable preload force ball screw system is shown in Figure 1, in which the master nut (connected to the worktable) and the slave nut are defined as Nut A and Nut B, respectively. Between Nut A and Nut B, three PEAs and three press force sensors are evenly distributed along the circumference of the sleeve. Due to the fact that the PEAs cannot withstand the shear force and tensile stress, it is our objective to design the system in such a way that the adjustable blots apply the initial preload force on the PEAs in axial direction to avoid the damage. In addition, these three parts are mounted evenly separated as depicted in the sectional view.

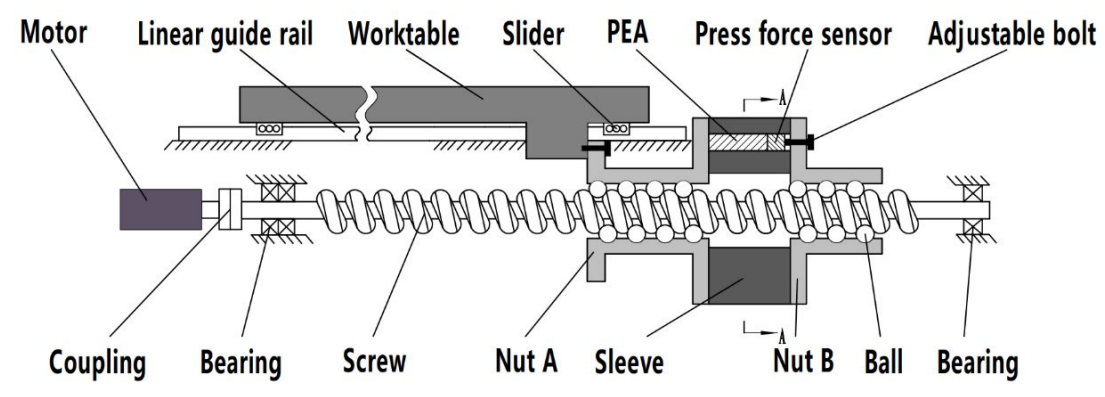

$(\mathbf{a})$

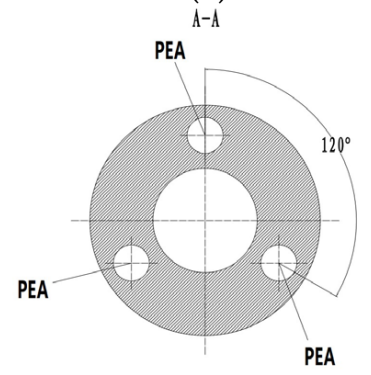

(b)

Figure 1. The schematic diagram of the variable preload force ball screw system: (a) overall configuration of the variable preload force ball screw system; (b) section view. 


\subsection{Force Analysis of Automatic Adjustable Preload System}

The ball screw system is used to realize $X$-axis motion, and the stiffness in the $X$ direction is the lowest [27]. As a result, the equivalent dynamic model of the system on the $\mathrm{X}$-axis is established as shown in Figure 2.

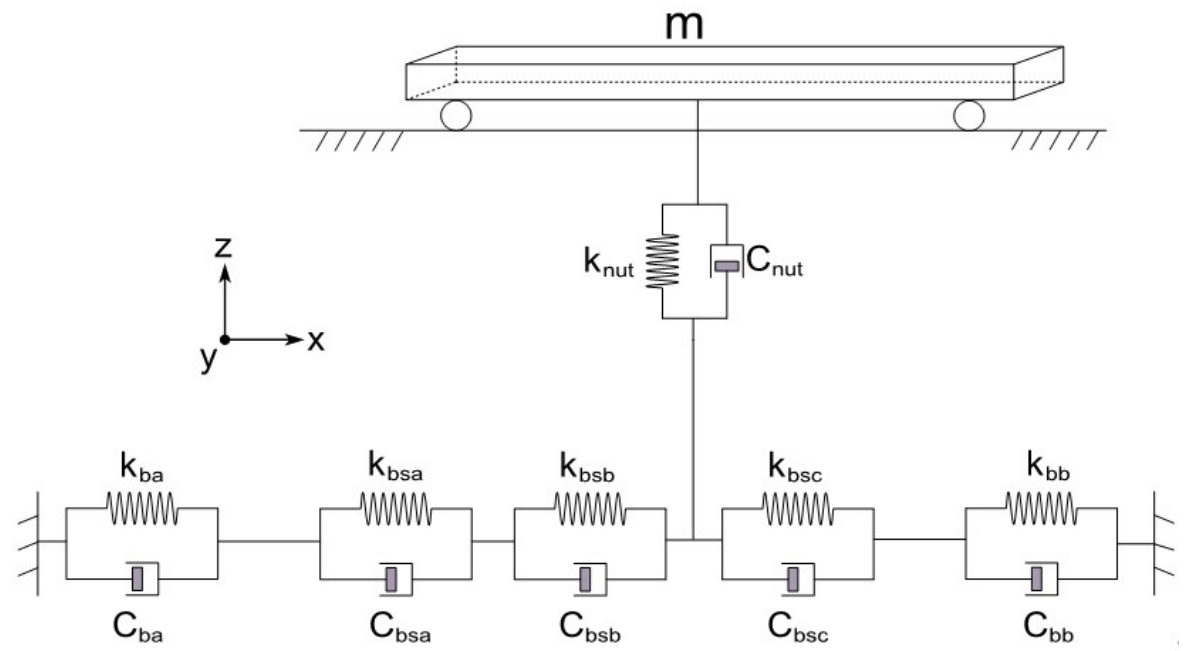

Figure 2. The equivalent dynamic model of the ball screw system.

In Figure 2, $k_{b a}, C_{b a}$ and $k_{b b}, C_{b b}$ are the equivalent stiffness and damping of the left and right bearing; $k_{b s a}, C_{b s a}, k_{b s b}, C_{b s b}$ and $k_{b s c}, C_{b s c}$ are the equivalent stiffness and damping of the left, middle and right sides of the screw; and $k_{n u t}$ and $C_{n u t}$ represent the equivalent stiffness and damping of the nuts.

In the model, the worktable is assumed to be a lumped mass element and the effect of servo stiffness is ignored. The dynamic equation of the system can be written as:

$$
m \ddot{x}+c \dot{x}+k x=0
$$

where $\ddot{x}, \dot{x}$ and $x$ are the acceleration, velocity and displacement of the system, respectively. $m$ is the total mass of the system, $k$ is the total stiffness coefficient. To simplify the analysis, damping (c) is not considered in this case.

Through applying voltage to the three PEAs simultaneously in real time, due to the inverse piezoelectric effect, the electrical energy will be converted into mechanical energy. Then, the preload force is applied to the ball screw. Consequently, the backlash is eliminated, and the stiffness of the mechanism is improved, the vibration is suppressed, and the resulting machining precision is enhanced. The force diagram of the preloaded system is presented in Figure 3. It shows that the actuators exert preload force on the slave nut and the master nut, bringing them into contact with the raceway of the screw, and the directions of the two contact angles are completely opposite.

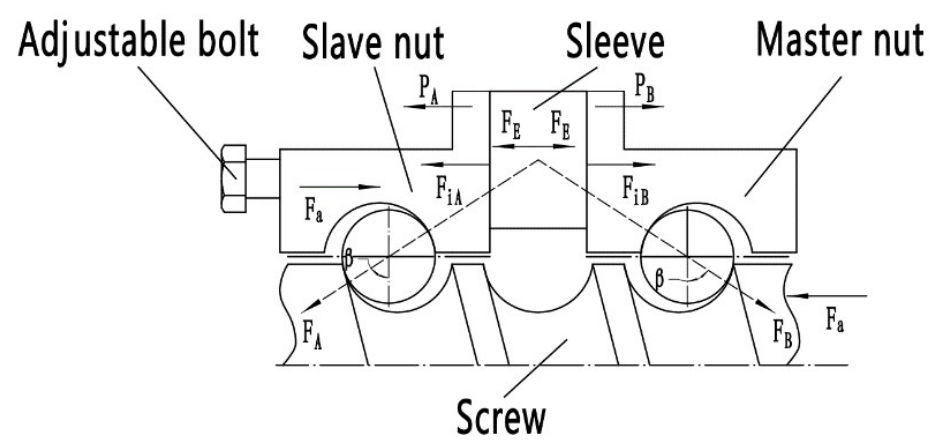

Figure 3. The force diagram of the preloaded system. 
As shown in Figure $3, F_{A}$ and $F_{B}$ are the normal force of a ball on the raceway in the slave nut and the master nut, respectively; $F_{E}$ is the preload force exerted by the PEAs in the sleeve of the structure; the initial preload force exerted by the adjustable bolt, $F_{i A}$ and $F_{i B}$, on the slave nut and the master nut, respectively, and the values of these are equal; $F_{a}$ is the axial load; $F_{p}$ is the total preload force. Furthermore, the ball number at work is $\mathrm{z}$. In addition, it is assumed that the structures of the slave nut and the master nut are the same.

When $F_{a}=0$, the initial preload force is the total preload force, and the formula can be written as:

$$
F_{p}=F_{i A}+F_{E}=F_{i B}+F_{E}=\mathrm{z} F_{A} \sin \beta \cos \alpha=\mathrm{z} F_{B} \sin \beta \cos \alpha
$$

The relationship between the normal force of a ball and the axial force, namely $F_{p}$, can be expressed as:

$$
F_{A}=F_{B}=\frac{F_{i A}+F_{E}}{\mathrm{z} \sin \beta \cos \alpha}=\frac{F_{i B}+F_{E}}{\mathrm{z} \sin \beta \cos \alpha}
$$

When $F_{a} \neq 0, P_{A}$ is the actual axial load on the slave nut, and $P_{B}$ is the actual axial load on the master nut, the following two equations can be obtained:

$$
P_{A}=F_{i A}+F_{E}+\Delta F \quad P_{B}=F_{i B}+F_{E}-\Delta F
$$

In this system, the two nuts are treated as having identical structure. Therefore, under the action of $F_{a}$, the force balance condition of the screw can be derived into Equations (5) and (6), below:

$$
\begin{gathered}
P_{A}=F_{a}+P_{B} \\
F_{a}-P_{A}+P_{B}=0
\end{gathered}
$$

From Equations (4)-(6), the formula is deduced as:

$$
2 \times \Delta F=F_{a}
$$

$+\Delta F$ and $-\Delta F$ are the force increment and decrement along with the change in $F_{a}$. Furthermore, $\alpha$ and $\beta$ are the helix angle and the contact angle of the ball screw, respectively.

Based on the condition of deformation coordination and mechanical superposition principle, the following equation holds [5]:

$$
\Delta d_{A}=\Delta d_{p}-\Delta d_{a}, \Delta d_{B}=\Delta d_{p}+\Delta d_{b}, \Delta d_{A}=\Delta d_{B}
$$

where $\Delta d_{A}, \Delta d_{B}, \Delta d_{p}, \Delta d_{a}, \Delta d_{b}$ are respectively the total axial contact deformation of the slave nut, the total axial contact deformation of the master nut, the axial contact deformation of ball screw under the preload force $F_{p}$ and the restoring deformation of the slave nut under the axial load $F_{a}$, the restoring deformation of the master nut under the axial load $F_{a}$.

Based on Hertz contact theory, the following formulas can be obtained:

$$
\Delta d_{p}=k\left(F_{p}\right)^{\frac{2}{3}}, \Delta d_{A}=k\left(F_{i A}+F_{E}-\Delta F\right)^{\frac{2}{3}}, \Delta d_{B}=k\left(F_{i B}+F_{E}+\Delta F\right)^{\frac{2}{3}}
$$

Equations (8) and (9) lead to the following relationships:

$$
1-\frac{\Delta F}{F_{p}}=\left(1-\frac{\Delta d_{a}}{\Delta d_{p}}\right)^{\frac{3}{2}}, 1+\frac{\Delta F}{F_{p}}=\left(1+\frac{\Delta d_{a}}{\Delta d_{p}}\right)^{\frac{3}{2}}
$$

From Equations (7)-(10), the following equation is yielded:

$$
\frac{F_{a}}{F_{p}}=\frac{3 \Delta d_{a}}{\Delta d_{p}}
$$


In [5], the formulas of $\Delta d_{a}$ can be obtained:

$$
\Delta d_{A}=\frac{1}{2}\left(\frac{3\left(F_{A}+\left(\frac{F_{a}}{2 z \sin \beta \cos \alpha}\right)\right.}{z E^{\prime}}\right)^{\frac{2}{3}} \cdot\left[\delta_{\dot{n}}\left(\sum \rho_{n}\right)^{\frac{1}{3}}+\delta_{s}\left(\sum \rho_{s}\right)^{\frac{1}{3}}\right](\cos \alpha)^{\frac{1}{3}}(\sin \beta)^{\frac{5}{3}}
$$

In Equation (12), $E^{\prime}$ stands for the equivalent modulus of elasticity, $\delta_{\dot{n}}=$ the dimensionless mechanical parameters of the nut, $\delta_{\dot{s}}=$ the dimensionless parameters of the nut, and $\sum \rho_{n}$ and $\sum \rho_{s}$ represent the sum of principal curvature of the nut and screw raceway. In addition, the axial contact stiffness of the double nut ball screw can be derived as:

$$
\mathrm{K}=\frac{3\left(2 F_{p}+F_{a}\right)^{\frac{1}{3}}}{\left(\frac{3}{2 z E^{\prime}}\right)^{\frac{2}{3}}\left[\delta_{\dot{n}}\left(\sum \rho_{n}\right)^{\frac{1}{3}}+\delta_{\dot{s}}\left(\sum \rho_{s}\right)^{\frac{1}{3}}\right](\cos \alpha)^{\frac{1}{3}}(\sin \beta)^{\frac{5}{3}}}
$$

From Equations (12) and (13), when the geometric parameters of the nut ball screw pair are fixed, its axial contact deformation and axial contact stiffness are mainly affected by the axial load and preload force.

\section{Control Model for Automatic Adjustable Preload System}

\subsection{Control Strategy of the PEAs}

Under practical conditions, the input-output characteristic curve shows the obvious nonlinear hysteresis error of PEAs. In the experiment, the initial preload force (the force applied by the adjust bolt) is $25 \mathrm{~N}$, and the measured curve can be seen in Figure 4 . In this work, the change in the curve shapes of the hysteresis loop under different external loading conditions is neglected. Considering the control strategy of the proposed structure, it is believed that the general linear control method is not able to meet the requirements of the system due to the nonlinear characteristic of the PEAs in this work. To achieve the feedforward compensation for the hysteresis error, a nonlinear hysteresis fitting model on the basis of a mathematical method needs to first be established, and the inverted model can be solved accordingly to obtain the relationship between the input voltage and the output force of the PEAs. This means that the more accurate the fitting model, the better the compensation effect; however, more parameters of the model need to be determined. As a result, a large amount of experimental data is required to identify the various parameters in the model. All in all, the above two methods have their own limitations: the feedforward control is greatly reliant on the accuracy of the modeling and there is a lack of resistance to unknown disturbances; moreover, the response time of the feedback control is relatively long.

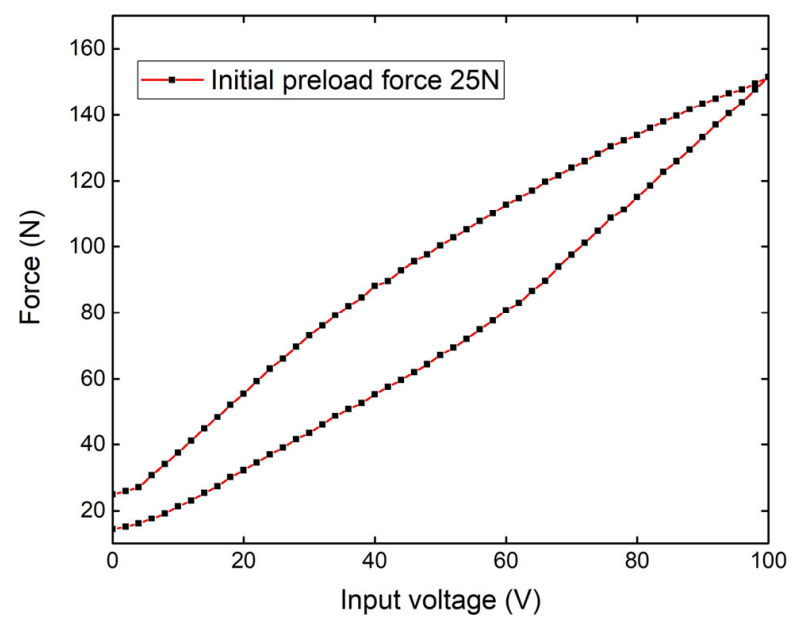

Figure 4. The curve of measured hysteresis loop. 


\subsection{The Modeling of Hysteresis}

The hysteretic model is an indispensable part of the preload force control that is necessary in order to compensate the hysteresis error. In addition, the P-I model has linear expression and low complexity, so it is relatively easy to obtain the parameters and the inverted model. In this paper, the P-I model is, consequently, used as the control model, and the play operator is regarded as the elementary operator. The operator is defined as follows: Set $t \in\left[t_{0}, t_{n}\right],\left(t_{0} \leq t_{1} \cdots t_{i-1} \leq t \leq t_{i} \cdots \leq t_{n-1} \leq t_{n}\right)$, when $t \in\left[t_{0}, t_{n}\right], x(t)$ changes monotonically. The play operator can be expressed as:

$$
y\left(t_{i}\right)=F\left(v(t), y\left(t_{i-1}\right), r\right)=\max \left\{v(t)-r, \min \left\{v(t)+r, y\left(t_{i-1}\right)\right\}\right\}
$$

where $y\left(t_{i}\right), v(t), y\left(t_{i-1}\right)$ and $r$ are the output of the operator at time $t_{i}$, the input, and the output at the previous moment and the threshold value, respectively. In addition, the initial operator $y_{0}$ is equal to 0 . The P-I hysteresis model can be built, based on the play operators with different threshold values, and its expression is as follows:

$$
\begin{gathered}
O(t)=\sum_{k=1}^{n} w_{k} F_{k}\left(v(t), y_{k}\left(t_{i-1}\right), r_{k}\right)=\sum_{k=1}^{n} \max \left\{v(t)-r_{k}, \min \left\{v(t)+r_{k}, y_{k}\left(t_{i-1}\right)\right\}\right\} \\
k=1,2, \cdots, n-1, n
\end{gathered}
$$

In Equation (15), $w_{k}$ and $r_{k}$ are the weight value and threshold value of the k-th play operator, and $0=r_{1}<\cdots r_{n}<+\infty, n$ is the number of operators.

The threshold value of the play operator is generally evenly distributed, so the value of the threshold can be determined by the range of the input voltage. The threshold value can be defined as:

$$
r_{k}=\frac{k-1}{n} v(t)_{\max }
$$

The value of $n$ is 40 , and $v(t)_{\max }$ is the maximum input voltage, which is $100 \mathrm{~V}$.

To obtain the parameters of the hysteretic model, first, the expression of the measured hysteresis curve is fitted; then, the curve is divided at each threshold value, and the corresponding weight value of each segment is deducted. Meanwhile, the slope of each segment is the sum of the weight values of all previous operators, with formula is given by:

$$
\left\{\begin{array}{c}
w_{1}=\frac{O_{v=r_{2}}-O_{v=r_{1}}}{r_{2}-r_{1}} \\
w_{2}+w_{1}=\frac{O_{v=r_{3}}-O_{v=r_{2}}}{r_{3}-r_{2}} \\
\vdots \\
w_{k}+w_{k-1}+\cdots+w_{2}+w_{1}=\frac{O_{v=r_{k+1}-O_{v=k}}}{r_{k+1}-r_{k}} \\
k=1,2,3, \cdots n-1, n \\
r_{k+1}=v(t)_{\max }
\end{array}\right.
$$

To simplify the calculation process of the model parameters, the input and output values are normalized. The cubic polynomial is used to fit the ascending section of the hysteresis curve. With the help of the Python algorithm, the expression of the curve can be obtained. In the same way, the descending segment can be generated. A comparison of the simulated hysteresis loop using the P-I model and the measured loop is shown in Figure 5. Furthermore, the curve expression is derived from Equation (18), where $w_{p k}$ represents the weight of the ascending segment, and $w_{q k}$ represents the weight of the descending segment. 
When the input voltage increases, $p$ takes the value 1 , and $q$ takes the value 0 . When the input voltage decreases, $p$ takes the value 0 and $q$ takes the value 1 .

$$
\begin{gathered}
O(t)=\sum_{k=1}^{n}\left(p w_{p k}+q w_{q k}\right) F_{k}\left(x(t), y_{k}\left(t_{i-1}\right), r_{k}\right) \\
=\sum_{k=1}^{n}\left(p w_{p k}+q w_{q k}\right) \max \left\{v(t)-r_{k}, \min \left\{v(t)+r_{k}, y_{k}\left(t_{i-1}\right)\right\}\right\} \\
\left\{\begin{array}{l}
p=1, q=0 v(t) \geq v\left(t_{i-1}\right) \\
p=0, q=1 v(t)<v\left(t_{i-1}\right)
\end{array}\right.
\end{gathered}
$$

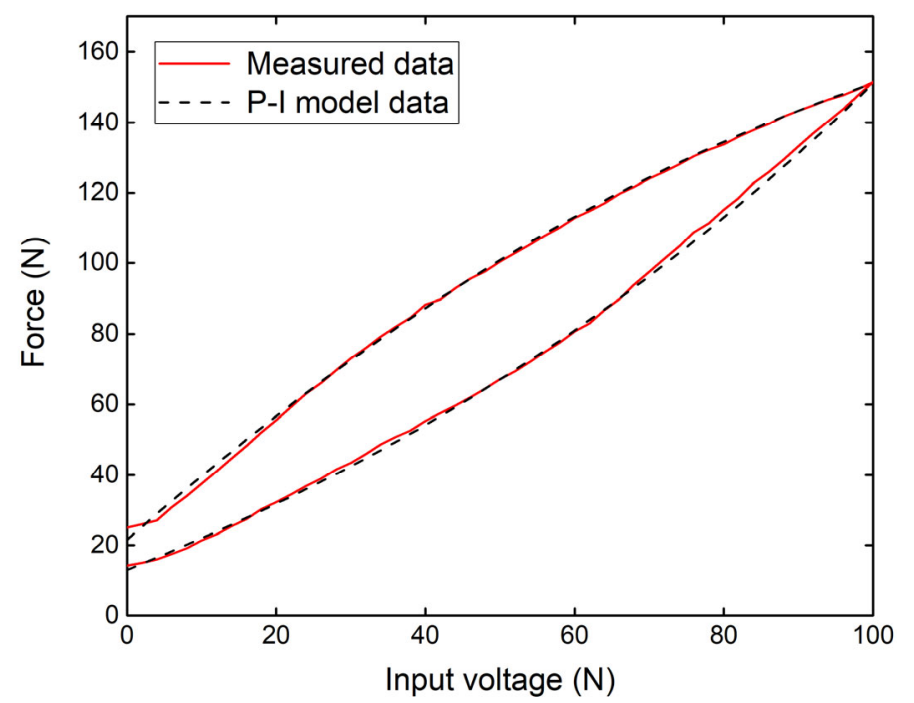

Figure 5. Comparison of the simulated hysteresis loop using the P-I model and the measured loop.

The inverted P-I model is symmetrical with the original model. In addition, the symmetry axis is a straight line $y=v$. The inverted model equation can be written as Equation (19), where $v(t)$ is the desired force, and $O^{-1}(t)$ refers to the corresponding input voltage. $w_{p k}^{-1}, w_{q k}^{-1}, r_{k}^{-1}$ are the inverse of their corresponding values, and their expression is shown in Equation (20). Through programming, the inverted model is obtained as illustrated in Figure 6.

$$
\begin{gathered}
O^{-1}(t)=\sum_{k=1}^{n}\left(p w_{p k}^{-1}+q w_{q k}^{-1}\right) F_{k}^{-1}\left(v(t), y_{k}\left(t_{i-1}\right), r_{k}^{-1}\right) \\
=\sum_{k=1}^{n}\left(p w_{p k}^{-1}+q w_{q k}^{-1}\right) \max \left\{v(t)-r_{k}^{-1}, \min \left\{v(t)+r_{k}^{-1}, y_{k}\left(t_{i-1}\right)\right\}\right\} \\
\left\{\begin{array}{l}
p=1, q=0 v(t) \geq v\left(t_{i-1}\right) \\
p=0, q=1 v(t)<v\left(t_{i-1}\right)
\end{array}\right. \\
\left\{\begin{array}{c}
w_{p 1}^{-1}=\frac{1}{w_{p 1}} \\
w_{p k}^{-1}=\frac{w_{q 1}^{-1}=\frac{1}{w_{q 1}}}{\left(\sum_{i=1}^{k} w_{p i}\right)\left(w_{p j}^{k-1} w_{p i}\right)} \\
w_{q k}^{-1}=\frac{-w_{q j}}{\left(\sum_{i=1}^{k} w_{q i}\right)\left(\sum_{i=1}^{k-1} w_{q i}\right)} \\
r_{k}^{-1}=\sum_{i=1}^{k} w_{p i}\left(r_{k}-r_{i}\right)
\end{array}\right.
\end{gathered}
$$




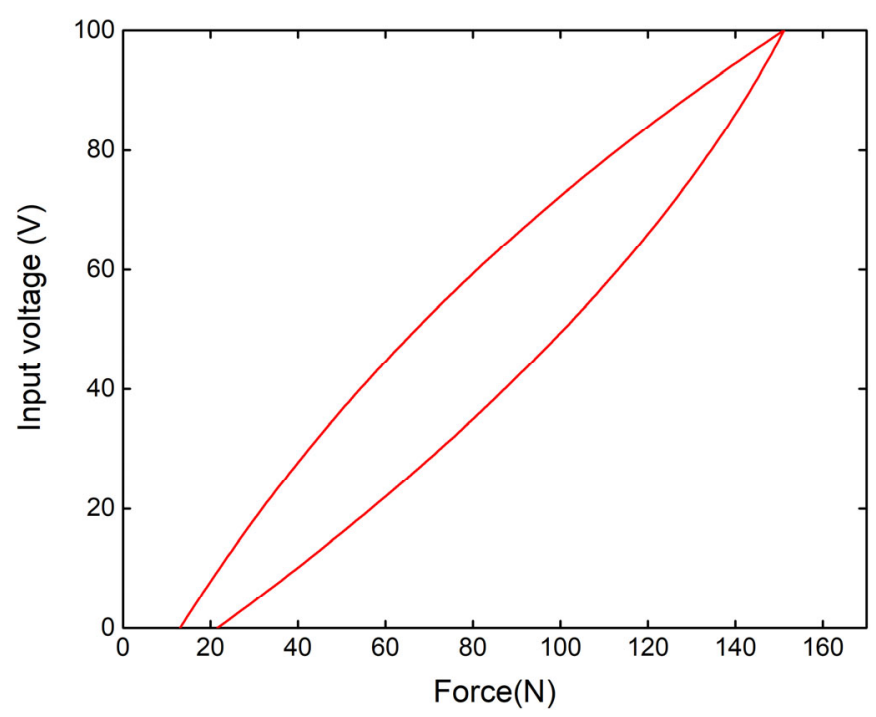

Figure 6. The inverted P-I model.

\section{Results of Experiments and Discussion}

In this section, the setup of the experimental rig is firstly introduced. After that, two control architectures are implemented on the variable preload force ball screw system. Finally, comparative experiments are conducted to verify the effectiveness of the controller.

\subsection{Experimental Setup}

The control system for the automatic adjustment of preload force is shown in Figure 7. To verify the effectiveness of the proposed control model, the experiments are conducted using three commercial PEAs (Physik Instrumente, Lederhose, Germany, P-888.91). In addition, the range of the input voltage is set between $0 \mathrm{~V}$ and $100 \mathrm{~V}$. The press force sensors (Kistler, Winterthur, Switzerland, Type 9313AA1) are respectively connected to the PEAs. Two I/O boards (Advantech, Taiwan, China, PCI-1716, PCI-1723) are used to transmit the data. The control model and data saving are programmed in Labview. The piezo driver is a low-cost amplifier for low-voltage PEAs. In addition, the system schematic connection is shown in Figure 8.

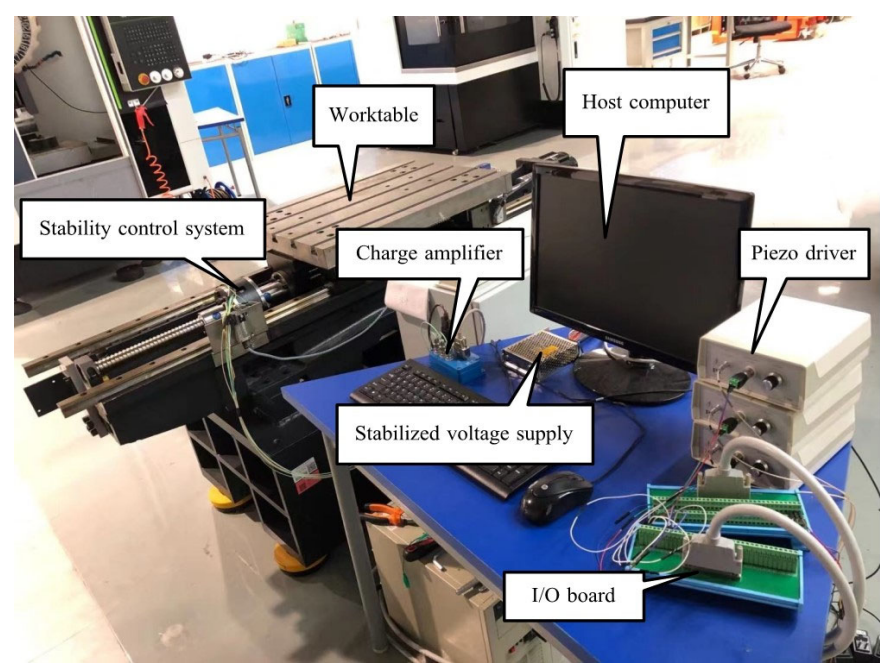

(a)

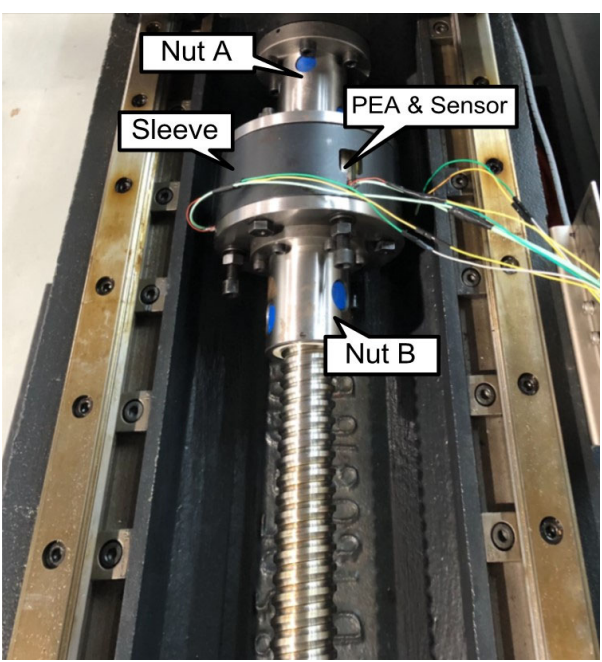

(b)

Figure 7. The experimental test rig: (a) overall configuration of the rig; (b) stability control system. 


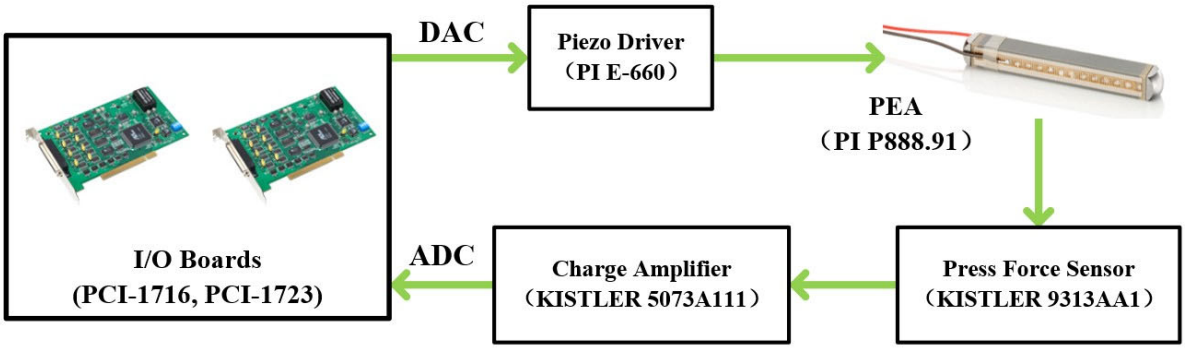

Figure 8. Schematics of system components.

\subsection{The Application of the Preload Ball Screw System with PID Control}

With the aim of evaluating the performance of the proposed feed drive system with respect to stability control, the traditional PID controller is used to verify the response of the structure with piezoelectric preloading actuators. Figure 9 shows a block diagram of the control system used for the force output control, which includes a classic PID controller, and a piezoelectric actuator model. The reason for selecting the PID control is to improve the transient response and steady-state error between the desired force output and the real-time force output.

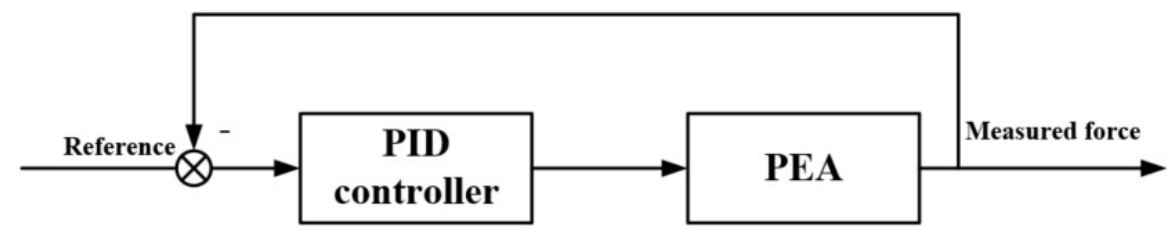

Figure 9. Block diagram of the PID controller.

In the experiment, the initial preload force is set as $25 \mathrm{~N}$, and the desired force is $60 \mathrm{~N}$. The PID controller gains used for the experiment are set initially with empirical tuning estimation to be $k_{p}=0.3, T_{i}=0.08, T_{d}=0.001$. The input voltage range of the closed loop force output control system is $0-110$ volts, and the tracking response of the system is shown in Figure 10. On the basis of the results of the test, the rise time, peak time and settling time are $1.69 \mathrm{~s}, 3.38 \mathrm{~s}$, and $3.38 \mathrm{~s}$, respectively, when the error of the response is selected to be $1.5 \mathrm{~N}$. Additionally, the tracking response of the step control with the trajectory (60 N-80 $\mathrm{N}-100 \mathrm{~N}-80 \mathrm{~N}-60 \mathrm{~N}$ ) is also tested, with results shown in Figure 11.

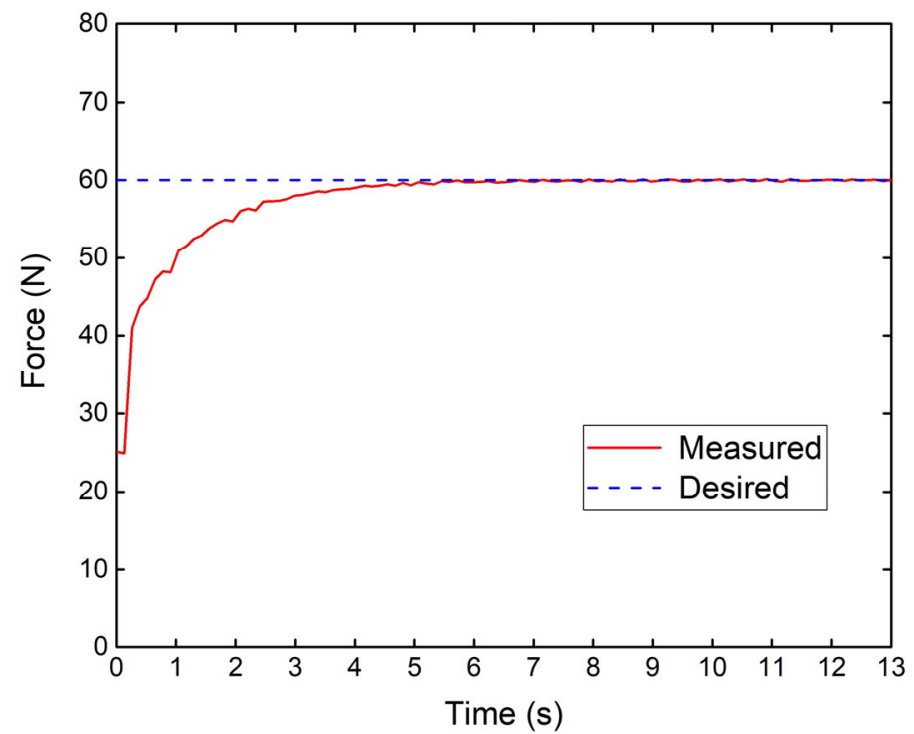

Figure 10. Tracking response curve of PID control. 


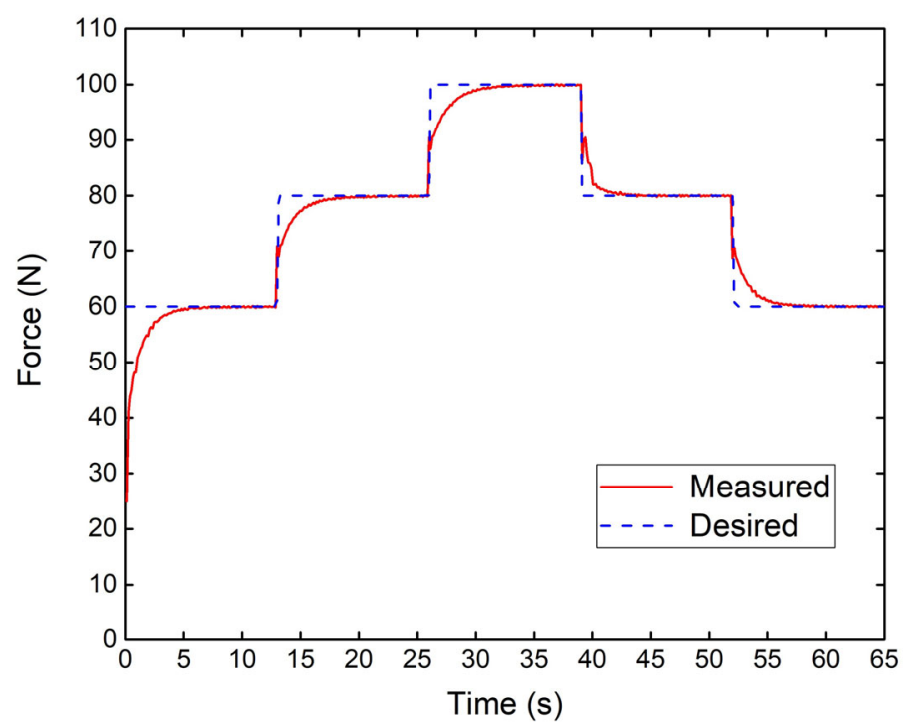

Figure 11. Tracking performance of PID control with a step-like trajectory.

\subsection{The Application of a Preload Ball Screw System with Feedforward Control and PID Control Strategy}

In practical applications, it is easily understood that the settling time of the control strategy has a significant effect on the feed drive system with respect to stability control, leading directly to better machining accuracy.

In this section, the P-I model-based feedforward control strategy is introduced, aiming at improving the feed drive stability response. The proposed block diagram of the P-I model feedforward control system is shown in Figure 12. The same working conditions and desired force output are set in the experiment in order to compare the feedforward control system with a classical PID controller. The force response of the system is shown in Figure 13. The results in Figure 13 reveal that the rise time, peak time and settling time are greatly shortened, to $0.08 \mathrm{~s}, 0.13 \mathrm{~s}$, and $1.43 \mathrm{~s}$, respectively. Compared to the former results without P-I model-based feedforward control, the rise time decreased by approximately $95 \%$, the peak time by approximately $96 \%$, and the settling time by approximately $58 \%$. This demonstrates that the performance of the machining tool with P-I model feedforward control is significantly improved. Similarly, the corresponding tracking performance with a step-like trajectory is also tested, with results shown in Figure 14, further verifying the effectiveness of the proposed feed drive stabilizing system.

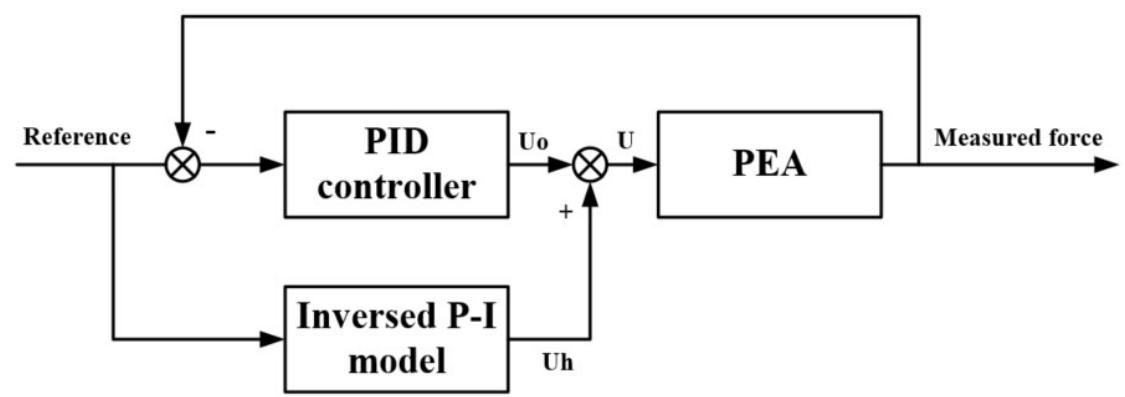

Figure 12. Block diagram of feedforward control and PID control.

On the basis of the aforementioned results of comparative experiments, it can be seen that the proposed control strategy has a higher efficiency. Therefore, the preload force is able to be adjusted accordingly, and the increase in stiffness and the enhancement of stability of the drive system can be achieved. All in all, this method has a bright future for use in cutting-edge equipment in the ultra-precision manufacturing industry. 


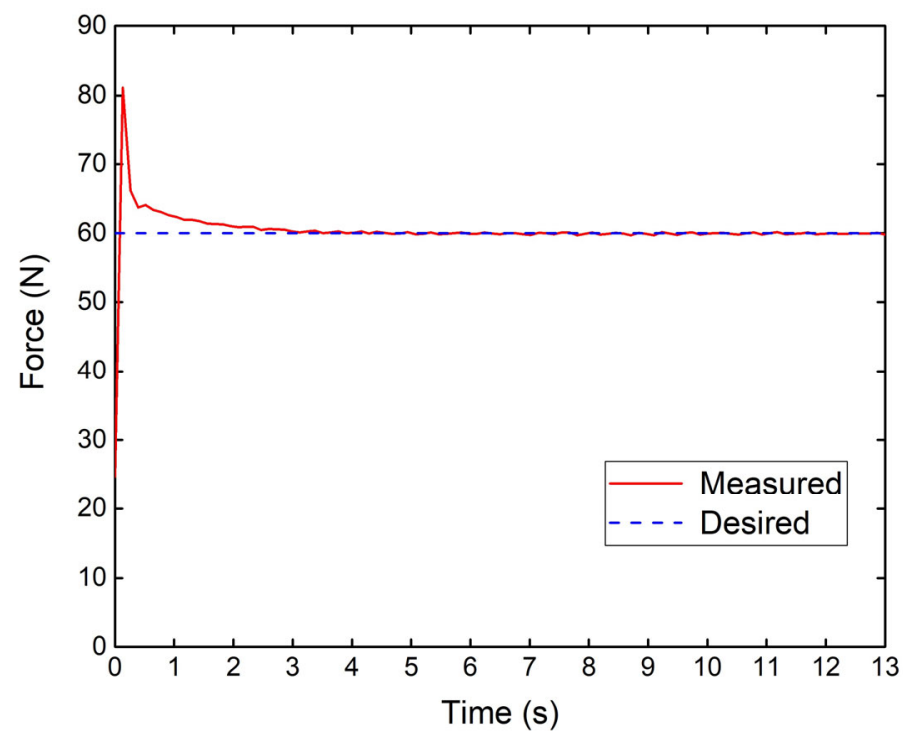

Figure 13. Step response curve of feedforward control and PID control.

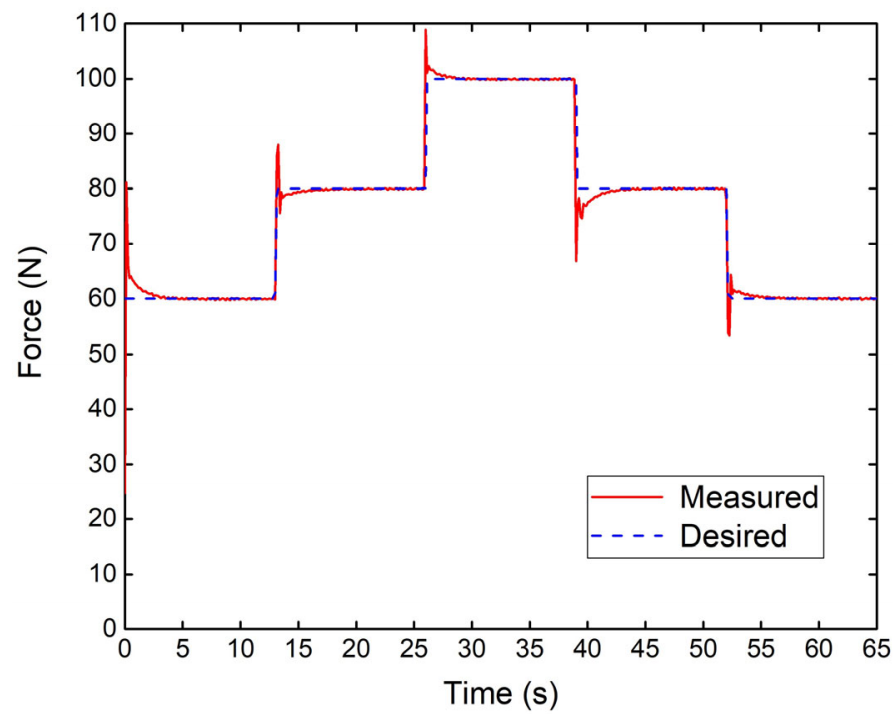

Figure 14. Tracking performance of feedforward control and PID control with a step-like trajectory.

In future, the optimization of the whole system will be discussed, and the criteria for designing preload feed drive systems will be discussed and analyzed in future studies. It is also believed that with the implementation of displacement measurement, the efficiency of the preload control system could also be improved.

\section{Conclusions}

In this paper, a variable preload force control device using three PEAs installed between the two nuts to exert preload forces on the ball screw in order to eliminate or reduce the backlash was developed to control the stability of the feed drive system by increasing the stiffness of the system at a high response rate using a P-I model-based control strategy. Considering the application of PEAs in a preload system, a force control system was designed and studied. By means of this methodology, the machining precision was significantly improved. Furthermore, the proposed control strategy, a feedforward compensator, and a PID controller were applied in the feed drive system, and the inverse of the P-I model was used as the feedforward compensator. On the basis of the model simulations and experimental testing, the high anticipated performance with respect to the stability of the feed drive system with the proposed P-I model-based control algorithm 
was proved to be superior to that obtained when using a conventional PID controller. By means of the proposed control strategy, the desired preload force can be adjusted with fast response and high precision compared to conventional strategies. In principle, this paper leads the initiative for future investigations in this area, with various feed drive system stability control strategies that could contribute directly to the attainment of ultra-precision machining.

Author Contributions: Conceptualization, G.Z. and H.J.; methodology, Y.-J.L.; software, H.J.; validation, G.Z., H.J. and Y.-J.L.; formal analysis, H.J.; investigation, G.Z.; resources, Y.-J.L.; data curation, Y.-J.L.; writing—original draft preparation, G.Z.; writing—review and editing, G.Z. and H.J.; visualization, Y.-J.L.; supervision, G.Z.; project administration, H.J.; funding acquisition, G.Z. All authors have read and agreed to the published version of the manuscript.

Funding: This research received no external funding.

Institutional Review Board Statement: Not applicable.

Informed Consent Statement: Not applicable.

Data Availability Statement: The data presented in this study are available on request from the corresponding author.

Acknowledgments: The authors acknowledge the editors and reviewers for their valuable comments and suggestions.

Conflicts of Interest: The authors declare no conflict of interest.

\section{References}

1. Quintana, G.; Ciurana, J. Chatter in machining processes: A review. Int. J. Mach. Tools Manuf. 2011, 51, 363-376. [CrossRef]

2. Pan, X.; He, X.; Wei, K.; Wu, H.; Gao, J.; Jiang, Z. Performance analysis and experimental research of electromagnetic-ring active balancing actuator for hollow rotors of machine tool spindles. Appl. Sci. 2019, 9, 692. [CrossRef]

3. Altintas, Y.; Verl, A.; Brecher, C.; Uriarte, L.; Pritschow, G. Machine tool feed drives. CIRP Ann. 2011, 60, 779-796. [CrossRef]

4. Frey, S.; Dadalau, A.; Verl, A. Expedient modeling of ball screw feed drives. Prod. Eng. 2012, 6, 205-211. [CrossRef]

5. Xu, X.R.; Song, X.C.; Jiang, H.K.; Li, Y.F. Influence Factors Comparison and Analysis of Axial Contact Stiffness between Single-nut and Double-nut Ball Screws. Adv. Mater. Res. 2014, 889-890, 555-558. [CrossRef]

6. Hu, G.; Zhang, D.; Gao, W.; Chen, Y.; Liu, T.; Tian, Y. Study on variable pressure/position preload spindle-bearing system by using piezoelectric actuators under close-loop control. Int. J. Mach. Tools Manuf. 2018, 125, 68-88. [CrossRef]

7. Paleu, V.; Gurau, G.; Comăneci, R.I.; Sampath, V.; Gurau, C.; Bujoreanu, L.-G. A new application of Fe-28Mn-6Si-5Cr (mass\%) shape memory alloy, for self-adjustable axial preloading of ball bearings. Smart Mater. Struct. 2018, 27, 075026. [CrossRef]

8. Kim, D.H.; Lee, C.M. A study on the development of a new conceptual automatic variable preload system for a spindle bearing. Int. J. Adv. Manuf. Technol. 2013, 65, 817-824. [CrossRef]

9. Gavrilin, A.N.; Moyzes, B.B.; Cherkasov, A.I.; Ditenberg, I.A.; Khairullin, A.R.; Glinenko, E.V. Pneumatic device of the preload and dynamic loads balancing to reduce the intensity of thermal processes in the metal cutting process. IOP Conf. Ser. Mater. Sci. Eng. 2017, 168, 012089. [CrossRef]

10. Cao, H.; Holkup, T.; Altintas, Y. A comparative study on the dynamics of high speed spindles with respect to different preload mechanisms. Int. J. Adv. Manuf. Technol. 2011, 57, 871. [CrossRef]

11. Hwang, Y.-K.; Lee, C.-M. A review on the preload technology of the rolling bearing for the spindle of machine tools. Int. J. Precis. Eng. Manuf. 2010, 11, 491-498. [CrossRef]

12. Lin, M.; Wang, Q.; Ju, X.; Fan, W. The research of double-nut ball screw preload based on GMA. In Proceedings of the 2016 13th International Conference on Ubiquitous Robots and Ambient Intelligence (URAI), Xi'an, China, 19-22 August 2016.

13. Hwang, Y.K.; Lee, C.M. Development of a newly structured variable preload control device for a spindle rolling bearing by using an electromagnet. Int. J. Mach. Tools Manuf. 2010, 50, 253-259. [CrossRef]

14. Ju, X.-J.; Lin, M.-X.; Fan, W.-T.; Bu, Q.-Q.; Wu, X.-J. Structure design and characteristics analysis of a cylindrical giant magnetostrictive actuator for ball screw preload. J. Cent. South Univ. 2018, 25, 1799-1812. [CrossRef]

15. Chen, J.; Dwang, I. A ballscrew drive mechanism with piezo-electric nut for preload and motion control. Int. J. Mach. Tools Manuf. 2000, 40, 513-526. [CrossRef]

16. Uchino, K. Piezoelectric Actuators and Ultrasonic Motors; Springer Science \& Business Media: Berlin, Germany, 1996 ; Volume 1.

17. Lok, M.; Helbling, E.F.; Zhang, X.; Wood, R.; Brooks, D.; Wei, G.-Y. A low mass power electronics unit to drive piezoelectric actuators for flying microrobots. IEEE Trans. Power Electron. 2017, 33, 3180-3191. [CrossRef]

18. Tuma, T.; Sebastian, A.; Lygeros, J.; Pantazi, A. The four pillars of nanopositioning for scanning probe microscopy: The position sensor, the scanning device, the feedback controller, and the reference trajectory. IEEE Control Syst. Mag. 2013, 33, 68-85. 
19. Jung, H.; Gweon, D.-G. Creep characteristics of piezoelectric actuators. Rev. Sci. Instrum. 2000, 71, 1896-1900. [CrossRef]

20. Gu, G.; Zhu, L.-M.; Su, C.-Y.; Ding, H. Motion control of piezoelectric positioning stages: Modeling, controller design, and experimental evaluation. IEEE/ASME Trans. Mechatron. 2012, 18, 1459-1471. [CrossRef]

21. Peng, J.; Chen, X. A survey of modeling and control of piezoelectric actuators. Mod. Mech. Eng. 2013, 3, 28248. [CrossRef]

22. Pop, N.; Caltun, O. Jiles-Atherton Magnetic Hysteresis Parameters Identification. Acta Phys. Polonica A 2011, 120, $491-496$. [CrossRef]

23. Smith, R.C.; Ounaies, Z. A domain wall model for hysteresis in piezoelectric materials. J. Intell. Mater. Syst. Struct. 2000, 11, 62-79. [CrossRef]

24. Song, G.; Zhao, J.; Zhou, X.; De Abreu-Garcia, J. Tracking control of a piezoceramic actuator with hysteresis compensation using inverse Preisach model. IEEE/ASME Trans. Mechatron. 2005, 10, 198-209. [CrossRef]

25. Mayergoyz, I.D.; Friedman, G. Generalized Preisach model of hysteresis. IEEE Trans. Magn. 1988, 24, 212-217. [CrossRef]

26. Kuhnen, K. Modeling, identification and compensation of complex hysteretic nonlinearities: A modified Prandtl-Ishlinskii approach. Eur. J. Control 2003, 9, 407-418. [CrossRef]

27. Zhang, J.; Zhang, H.; Du, C.; Zhao, W. Research on the dynamics of ball screw feed system with high acceleration. Int. J. Mach. Tools Manuf. 2016, 111, 9-16. [CrossRef] 